\title{
On the accessible points in the Julia sets of some entire functions
}

by

\author{
Bogusława Karpińska (Warszawa)
}

\begin{abstract}
We prove that for some families of entire functions whose Julia set is the complement of the basin of attraction every branch of a tree of preimages starting from this basin is convergent.
\end{abstract}

1. Introduction. We consider a class of entire maps on the complex plane which includes the functions described by Devaney and Tangerman in [5] which admit a Cantor bouquet in their Julia sets. The simplest examples of such maps are $\lambda \exp z, \lambda \sin z, \lambda \cos z$. Another class of interesting examples is described in [12] and [13].

Let $E$ be a transcendental entire function such that

(1) its asymptotic and critical values are contained in a bounded set.

Let $D$ be a closed topological disk in $\mathbb{C}$ containing all the critical and asymptotic values of $E$ in its interior and let $\Gamma$ be the complement of $D$. Denote by $T$ a connected component of $E^{-1}(\Gamma)$. The set $T$ is called an exponential tract; it is a topological disk whose closure contains infinity and $E_{\mid T}$ is an infinite degree covering (see [5]). There can be finitely or infinitely many exponential tracts. Let $T_{r}(r=0,1 \ldots)$ be the exponential tracts for $E$.

We shall assume that

(2) $0 \in D$ and every exponential tract satisfies $T_{r} \cap D=\emptyset$.

Then $E$ has an attracting fixed point in $D$ whose basin of attraction $A$ contains $D$. We shall also assume that

(3) the Julia set is the complement of the basin of attraction $A$.

2000 Mathematics Subject Classification: Primary 37F10, 30D05.

Partially supported by Polish KBN Grant No. 2 PO3A 00917 and PW Grant No. 504 G11 200003000. 
If $E$ is critically finite (i.e. has finitely many critical and asymptotic values) then (3) is satisfied because $E$ has neither wandering domains nor Baker domains (see [6] and [1]).

We shall denote by $\mathcal{F}$ the class of entire functions satisfying (1)-(3). Let $\gamma:[0, \infty) \rightarrow \widehat{\mathbb{C}}$ be a smooth curve disjoint from the union of the exponential tracts and such that $z=\gamma(0) \in D, \gamma(0, \infty) \cap D=\emptyset$, and $\lim _{t \rightarrow \infty} \gamma(t)$ $=\infty$. We use this curve to define the fundamental domains in every $T_{r}$ by taking components of the preimage of $\Gamma \backslash \gamma[0, \infty)$. Denote by $\gamma_{i}^{r}, i \in \mathbb{Z}$, the components of $E^{-1}(\gamma[0, \infty))$ so that $\gamma_{i}^{r}$ and $\gamma_{i+1}^{r}$ bound one fundamental domain $P_{i}^{r}$ (see Fig. 1).

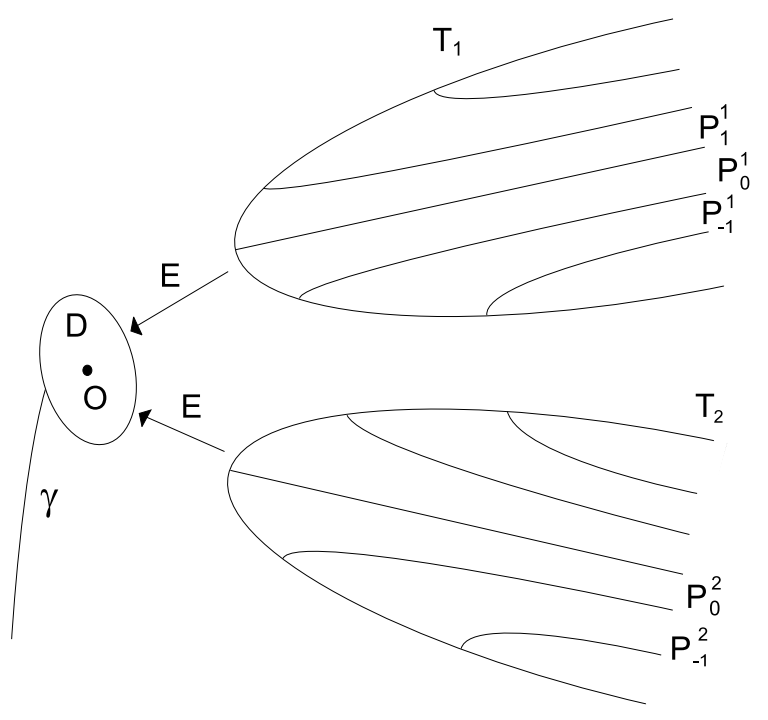

Fig. 1. Exponential tracts and fundamental domains

It is proved in [5] that under certain assumptions concerning the growth of $E$ in each $T_{r}$, for $E$ critically finite, the set

$$
\Lambda_{N}=\left\{z: E^{j}(z) \in \bigcup_{i=-N}^{N} P_{i}^{r} \text { for all } j \geq 0\right\}
$$

is homeomorphic to the Cantor $N$-bouquet which we define below.

For a positive integer $N$ we consider the space of sequences of integers between $-N$ and $N$ :

$$
\Sigma_{N}=\left\{\left(s_{0}, s_{1}, \ldots\right): s_{j} \in \mathbb{Z},\left|s_{j}\right| \leq N\right\} .
$$

The set $\Sigma_{N}$ is homeomorphic to a Cantor set. Define $\sigma: \Sigma_{N} \rightarrow \Sigma_{N}$ by $\sigma\left(s_{0}, s_{1}, s_{2}, \ldots\right)=\left(s_{1}, s_{2}, \ldots\right)$. We call a closed subset $C_{N}$ of $\mathbb{C}$ a Cantor $N$-bouquet for an entire function $f$ if $f\left(C_{N}\right) \subset C_{N}$ and there exists a homeomorphism $h: \Sigma_{N} \times[0, \infty) \rightarrow C_{N}$ with the following properties: 
(a) $\left(\pi \circ h^{-1} \circ f \circ h\right)(s, t)=\sigma(s)$ for all $t \in[0, \infty)$, where $\pi: \Sigma_{N} \times[0, \infty)$ $\rightarrow \Sigma_{N}$ is the projection $\pi(s, t)=s$.

(b) $\lim _{t \rightarrow \infty} h(s, t)=\infty$.

(c) $\lim _{n \rightarrow \infty} f^{n}(h(s, t))=\infty$ if $t>0$.

The simplest example of a Cantor bouquet (i.e. $\mathcal{C}=\overline{\bigcup_{N=1}^{\infty} C_{N}}$ ) arising in the dynamics of entire maps is the Julia set for the map $E_{\lambda}(z)=\lambda e^{z}$, where $z \in \mathbb{C}$ and $\lambda \in(0,1 / e)$, described in detail in [4] and [5]. The function $E_{\lambda}$ has two real fixed points, one of them attracting, denote it by $p_{\lambda}$, and the other repelling, denote it by $q_{\lambda}$. Choose $\nu_{\lambda}$ such that $\nu_{\lambda}<q_{\lambda}$ and if $\operatorname{Re} z \geq \nu_{\lambda}$ then $\left|E_{\lambda}^{\prime}(z)\right|>1$, and denote by $H$ the half-plane $\left\{z: \operatorname{Re} z \geq \nu_{\lambda}\right\}$. This is the only exponential tract $T$. The left half-plane $\mathbb{C} \backslash H$ is mapped by $E_{\lambda}$ into itself, it is contained in the basin of attraction. The Julia set of $E_{\lambda}$ is contained in $H$ ( $H$ is the only exponential tract). We divide $H$ into infinitely many strips: for $k \in \mathbb{Z}$,

$$
P(k)=\left\{z \in \mathbb{C}: \operatorname{Re} z \geq \nu_{\lambda},(2 k-1) \pi \leq \operatorname{Im} z<(2 k+1) \pi\right\} .
$$

This way we get fundamental domains (the half-line $\left\{z: \operatorname{Re} z<-E_{\lambda}\left(\nu_{\lambda}\right)\right.$, $\operatorname{Im} z=0\}$ plays the role of $\gamma$ ). If the forward orbit of $z$ is completely contained in $H$ then the itinerary of $z$ is defined to be the sequence $s=$ $\left(s_{0}, s_{1}, \ldots\right)$ such that $s_{j}=k$ if $E_{\lambda}^{j}(z) \in P(k)$.

The set of points which have a given sequence $s$ as itinerary either forms a curve $X_{s}$ lying in the Julia set $J_{\lambda}$ or is empty in $\mathbb{C}$. Each $X_{s}$ is the image of a continuous embedding $\phi_{s}:[0,1) \rightarrow \mathbb{C}$ with $\phi_{s}(t) \rightarrow \infty$ as $t \rightarrow 1$. The set $J_{\lambda} \backslash \infty$ is a disjoint union of curves $X_{s}$ called hairs. Devaney and Goldberg [3] proved that the point $z_{s}=\phi_{s}(0)$ is accessible from the basin of attraction (i.e. there exists a path $\beta:[0,1) \rightarrow \Omega_{\lambda}$ such that $\lim _{t \rightarrow 1} \beta(t)=z_{s}$ ). The points $z_{s}$ can be obtained as the limit points of the branches of the tree of preimages which we describe below; moreover all branches converge to points $z_{s}$ or to infinity. Devaney and Goldberg also proved that $z_{s}$ is the unique point in $X_{s}$ accessible from the basin of attraction. It is shown in [8] and [9] that the set of accessible points for $E_{\lambda}$ has Hausdorff dimension 2 while the rest of the Julia set (i.e. hairs without their endpoints) has Hausdorff dimension 1 . We expect that in the class $\mathcal{F}$ the structure of the Julia set is similar (under some additional assumptions). In particular this leads to the question whether the Hausdorff dimension of the set of accessible points equals the dimension of the whole Julia set. Here we make a first step towards an answer: we prove the convergence of all branches in $\widehat{\mathbb{C}}$.

In this paper we assume the following:

(i) For a given exponential tract $T_{r}$ all the fundamental domains extend to infinity in the same direction, i.e. for all $j \in \mathbb{Z}, \gamma_{j}^{r}$ are $C^{1}$-asymptotic to a straight line with fixed direction $\theta^{r}$. Moreover we assume that there 
exists a constant $c$ such that for every $r$ and every $j \in \mathbb{Z}$, if $z \in P_{j}^{r}$ then $\operatorname{dist}\left(z, l_{\theta^{r}}\right)<c$, where dist means Euclidean distance and $l_{\theta^{r}}$ is a straight line with direction $\theta^{r}$.

(ii) There exist constants $R>0$ and $\varepsilon<\pi / 2$ such that for every branch $G$ of $E^{-1}$ and for any $z_{1}, z_{2}$ such that $E\left(z_{1}\right), E\left(z_{2}\right)$ lie in the same fundamental domain and $\left|E\left(z_{1}\right)\right|,\left|E\left(z_{2}\right)\right|>R$ the following inequality holds:

$$
\left|\operatorname{Arg} G^{\prime}\left(E\left(z_{1}\right)\right)-\operatorname{Arg} G^{\prime}\left(E\left(z_{2}\right)\right)\right|<\varepsilon
$$

(iii) There exists a constant $K$ such that $\operatorname{dist}(D, T)>K$ for every exponential tract $T$.

The Julia set of $E$ consists precisely of those points which remain in $\bigcup_{r} T_{r}$ under iteration of $E$ (because we assume that the Julia set is the complement of the basin of attraction).

For every $z \in J(E)$ we define the itinerary of $z$ to be the sequence of symbols $s(z)=\left(\varepsilon_{0}, s_{0}, \varepsilon_{1}, s_{1}, \ldots, \varepsilon_{n}, s_{n}, \ldots\right)$, where $s_{i} \in \mathbb{Z}$ and $\varepsilon_{i} \in \mathbb{N}$ if the number of exponential tracts is infinite, while $\varepsilon_{i} \in\{0, \ldots, p\}$ if there are $p+1$ exponential tracts, such that

$$
\varepsilon_{i}=t \quad \text { and } \quad s_{i}=j \quad \text { if } E^{i}(z) \in P_{j}^{t} .
$$

We use double coding to distinguish fundamental domains in different tracts.

We will consider the tree of preimages for $E$ (introduced in [7], see also [11]) defined as follows. Fix $z_{0} \in A \backslash D$ such that $\frac{1}{2} K<\operatorname{dist}\left(z_{0}, D\right)<K$. The preimage of $\left\{z_{0}\right\}$ is contained in the union of exponential tracts; for each $r \in \mathbb{N}$ and $j \in \mathbb{Z}$ there is exactly one $z_{j}^{r} \in P_{j}^{r}$ such that $E\left(z_{j}^{r}\right)=z_{0}$.

Consider arcs $\beta_{j}^{r}:[0,1] \rightarrow A \backslash D$ joining $z_{0}$ to the corresponding $z_{j}^{r}$, $j \in \mathbb{Z}$ (pairwise disjoint except for $\beta_{j}^{r}(0)$ ). They can be chosen such that $\beta_{j}^{r} \cap\{z: \operatorname{dist}(z, D)<K / 2\}=\emptyset$. For a given sequence of symbols $s=$ $\left(\varepsilon_{0}, s_{0}, \varepsilon_{1}, s_{1}, \ldots, \varepsilon_{n}, s_{n}, \ldots\right)$ we define $\beta_{0}(s)=\beta_{s_{0}}^{r}$ and $\beta_{n}(s)$ by induction: Suppose we have already defined $\beta_{i}:[i, i+1] \rightarrow A \backslash D$ for $0 \leq i \leq n$ in such a way that for $i \geq 1, \beta_{i}(s)(i)=\beta_{i-1}(s)(i)$ and $E\left(\beta_{i}(s)\right)=\beta_{i-1}(\sigma(s))$, where $\sigma$ is the shift map $\sigma\left(\varepsilon_{0}, s_{0}, \varepsilon_{1}, s_{1}, \ldots, \varepsilon_{n}, s_{n}, \ldots\right)=\left(\varepsilon_{1}, s_{1}, \ldots, \varepsilon_{n}, s_{n}, \ldots\right)$. Then we define $\beta_{n+1}(s)$ to be the curve parameterized on $[n+1, n+2]$ such that

$$
\beta_{n+1}(s)(n+1)=\beta_{n}(s)(n+1), \quad E\left(\beta_{n+1}(s)\right)=\beta_{n}(\sigma(s)) .
$$

Joining the curves $\beta_{n}(s)$ for $n \in \mathbb{N}$ we obtain the curve $\beta(s)$ parameterized on $[0, \infty]$ which is called the branch of the tree of preimages for a given itinerary $s$. The curve $\beta(s)$ is contained in the basin of attraction. If $\beta(s)$ is convergent then its limit point belongs to the boundary of $A$ (that is, $J(E)$ ) or it is infinity.

In the next section we shall make one more assumption concerning the behavior of the branches $\beta_{0}$ of the first generation. 
The purpose of this paper is to prove that under the above assumptions the following holds:

TheOREM. Every branch of the tree of preimages is convergent in $\widehat{\mathbb{C}}$.

In particular, this means that for every itinerary $s$ there exists at least one accessible point in $J(E) \cup\{\infty\}$ whose itinerary is $s$.

EXAmples. 1. The example described above, $E_{\lambda}(z)=\lambda \exp z$, where $\lambda \in(0,1 / e)$, satisfies all our assumptions.

2. Now consider the function $S_{\lambda}(z)=\lambda\left(e^{z}-e^{-z}\right) / 2$, where $\lambda \in(0,1)$, i.e. $\lambda \sin z$ after a change of coordinates. It has one attracting fixed point at 0 and two real repelling fixed points with the same modulus $q$. The vertical lines $\{z: \operatorname{Re} z=\nu\}$ and $\{z: \operatorname{Re} z=-\nu\}$, where $0<\nu<q$, are mapped onto an ellipse with center at 0 which intersects the real axis at $S_{\lambda}(\nu)<\nu$. The half-planes $\{z: \operatorname{Re} z>\nu\}$ and $\{z: \operatorname{Re} z<-\nu\}$ are mapped onto the exterior of this ellipse. These half-planes are exponential tracts for $S_{\lambda}$. Critical values of $S_{\lambda}$ are attracted to 0 and there are no asymptotic values. The Julia set for $S_{\lambda}$ contains of a pair of Cantor bouquets, one tending to $\infty$ in the direction of the positive real axis and the other in the direction of the negative real axis (see [5]).

3. Consider the function defined by

$$
f(z)=\frac{1}{2 \pi i} \int_{L} \frac{\exp (\exp t)}{t-z} d t,
$$

where $L$ is the boundary of the region

$$
G=\{z: \operatorname{Re} z>0,-\pi<\operatorname{Im} z<\pi\}
$$

described in the clockwise direction, and $z \in \mathbb{C} \backslash \bar{G}$. The function $f$ can be analytically continued to a transcendental entire function; denote it also by $f$. This function was investigated by Stallard [12] who proved that the Julia sets for the functions $f_{K}(z)=f(z)-K$ for $K$ sufficiently large have Hausdorff dimension arbitrarily close to 1 . It turns out (see [12]) that there exists a constant $c$ such that $|f(z)|<c$ for $z \in \mathbb{C} \backslash G$, hence for large $K$, $f_{K}$ maps $\mathbb{C} \backslash G$ into a bounded subset of the left half-plane $\{z: \operatorname{Re} z<0\}$. Moreover, for $z \in G$,

$$
f(z)=\exp \left(e^{z}\right)+O(1 / z) \quad \text { as } z \rightarrow \infty .
$$

All critical and asymptotic values of $f_{K}$ are contained in a bounded subset of the left half-plane. Let $D$ be a topological disk in the left half-plane containing all critical and asymptotic values. Since $D$ is mapped into itself there exists an attracting fixed point in $D$. It follows from [2] that $f_{K}$ has no wandering domains. Hence the basin of attraction is the only component of the Fatou set. All exponential tracts for $f_{K}$ are contained in $G$; there are infinitely many of them. 
2. Convergence of the branches in the tree of preimages. Let $z_{1}, z_{2}$ be points belonging to the same exponential tract $T$ with asymptotic direction $\theta$. We will say that $z_{1}$ precedes $z_{2}$ and write $z_{1} \prec z_{2}$ if $\left|\operatorname{Re}\left(L_{\theta}\left(z_{1}\right)\right)\right| \leq\left|\operatorname{Re}\left(L_{\theta}\left(z_{2}\right)\right)\right|$, where $L_{\theta}$ denotes rotation through the angle $-\theta$. Similarly one can define the relation $\prec$ in the case that only one of the points $z_{1}, z_{2}$ belongs to an exponential tract: if $z_{1} \notin \bigcup_{j} T_{j}$ and $z_{2} \in T_{i}$ for some $i$ then we say that $z_{1} \prec z_{2}$ if $\operatorname{Dist}\left(z_{1}, 0\right) \leq \operatorname{Dist}\left(T_{i}, 0\right)$, where Dist denotes the spherical metric in $\widehat{\mathbb{C}}$. In what follows, Diam denotes diameter in the spherical metric.

Our aim is to prove

THEOREM 2.1. Let $E$ be a transcendental entire function in the class $\mathcal{F}$ satisfying assumptions (i)-(iii). Assume that there exists $j$ such that for every itinerary s,

$$
\text { if } a \in \beta(s)_{\mid[0,1)}, \quad b \in \beta(s)_{\mid[j, \infty)} \quad \text { then } a \prec b .
$$

Then for every itinerary s the branch $\beta(s)$ is convergent (to a point in $J(E)$ $\cup\{\infty\})$.

The proof of the theorem is based on the following observation:

Proposition 2.2. Let $E$ be a transcendental entire function in the class $\mathcal{F}$ satisfying (i)-(iii). Then there exists $C>0$ such that for every $x \in \Gamma$ with $\operatorname{dist}(x, D)>\frac{1}{2} K$, every $y \in \bigcup_{r} T_{r}$ and every branch $G$ of the inverse function for $E$, if $x \prec y$ and $G(y) \prec G(x)$ then

$$
\min \{\operatorname{Dist}(G(x), \infty), \operatorname{Dist}(G(y), \infty)\} \geq C \operatorname{Dist}(G(x), G(y)) .
$$

Proof. Let $\alpha:[0, \infty) \rightarrow \widehat{\mathbb{C}}$ be a curve joining $x$ to infinity and containing $y$ such that $\alpha(0)=x$ and such that there exists $t_{0}$ with $\alpha_{\mid\left[t_{0}, \infty\right)}$ contained in one fundamental domain and close to a straight line (assumption (i)). Then $G(\alpha)$ is contained in some exponential tract and it tends to infinity (because $E$ has no poles). The condition $G(y) \prec G(x)$ means that $G(\alpha)$ turns back in the part between $x$ and $y$.

If $\alpha \subset\{z:|z|>R\}$ then it follows from (ii) that

$$
|G(x)-G(y)|<c^{\prime},
$$

where $c^{\prime}$ is close to $c$, the "width" of the fundamental domain. But this means that there exists $C>0$ such that

$$
\min \{\operatorname{Dist}(G(x), \infty), \operatorname{Dist}(G(y), \infty)\} \geq C \operatorname{Dist}(G(x), G(y)) .
$$

If $\alpha$ is not contained in $\{z:|z|>R\}$ then we can choose a point $z=\alpha\left(t_{z}\right)$ such that $|z| \leq R$ and

$$
\operatorname{Dist}(G(x), G(y)) \leq \operatorname{Dist}(G(x), G(z))
$$


(if $|y|<R$ then we take $z=y$ ). Let $M$ be a topological disk containing $x$ and $z$ such that $M \subset B(0, R)$ and $\operatorname{Dist}(M, D)>\frac{1}{2} K$. Let

$$
\begin{aligned}
N & =\left\{z: \forall m \in M \operatorname{Dist}(z, m)<\frac{1}{4} K\right\}, \\
N^{\prime} & =\left\{z: \forall m \in M \operatorname{Dist}(z, m)<\frac{1}{2} K\right\} .
\end{aligned}
$$

Since $N^{\prime}$ does not meet $D$ we may use the spherical version of the Koebe Distortion Theorem (see [10, Lemma 1.2]). Take any $w \in \partial N$. Then there exists a constant $L$ such that

$$
\frac{\operatorname{Dist}(G(x), G(z))}{\operatorname{Dist}(G(x), G(w))} \leq L \frac{\operatorname{Dist}(x, z)}{\operatorname{Dist}(x, w)} .
$$

But the ratio $\operatorname{Dist}(x, z) / \operatorname{Dist}(x, w)$ is bounded from above for all $w$ so it follows that there exists $C>0$ such that for an appropriate $w$,

$$
\begin{aligned}
\operatorname{Dist}(G(x), \infty) & \geq \operatorname{Dist}(G(x), G(w)) \geq C \operatorname{Dist}(G(x), G(z)) \\
& \geq C \operatorname{Dist}(G(x), G(y)) .
\end{aligned}
$$

Since $G(x) \prec G(y)$ we also have

$$
\operatorname{Dist}(G(y), \infty) \geq C \operatorname{Dist}(G(x), G(y))
$$

Now we give the proof of the theorem:

Proof of Theorem 2.1. Assume that for some itinerary $s$ the branch $\beta=\beta(s):[0, \infty) \rightarrow \mathbb{C}$ of the tree of preimages has two different limit points $x$ and $y$. Let $y \prec x$. Then one can choose two sequences of points in $\beta: x_{n}=\beta\left(u_{n}\right), y_{n}=\beta\left(v_{n}\right), u_{n}, v_{n} \in[0, \infty), x_{n} \rightarrow x, y_{n} \rightarrow y$ as $n \rightarrow \infty$, such that the pair $\left(x_{n}, y_{n}\right)$ lies in reverse order for every $n$, that is,

$$
y_{n} \prec x_{n}, \quad u_{n}<v_{n}, \quad \text { for } n=1,2, \ldots
$$

We may assume that for every $n, x_{n} \in \beta_{i_{n}}$ and $y_{n} \in \beta_{j_{n}}$, where $i_{n} \neq j_{n}$. By our assumptions for every $n$ there exists $t_{n} \in \mathbb{N}$ such that

$$
E^{t_{n}}\left(x_{n}\right) \prec E^{t_{n}}\left(y_{n}\right), \quad E^{t_{n}^{\prime}}\left(y_{n}\right) \prec E^{t_{n}^{\prime}}\left(x_{n}\right) \quad \text { for every } t_{n}^{\prime}<t_{n} .
$$

Note that $t_{n} \neq 0$ (because $x_{n}$ and $y_{n}$ are in reverse order). Moreover at least one of $E^{t_{n}}\left(x_{n}\right), E^{t_{n}}\left(y_{n}\right)$ belongs to an exponential tract. Then by Proposition 2.2 ,

$$
\begin{aligned}
\min \left\{\operatorname{Dist}\left(E^{t_{n}-1}\left(x_{n}\right), \infty\right), \operatorname{Dist}\left(E^{t_{n}-1}\left(y_{n}\right), \infty\right)\right\} \\
\geq C \operatorname{Dist}\left(E^{t_{n}-1}\left(x_{n}\right), E^{t_{n}-1}\left(y_{n}\right)\right) .
\end{aligned}
$$

Now we consider two cases:

CASE I: $t_{n} \rightarrow \infty$. Let $m_{n}=t_{n}-1$. By the above inequality for every $n$ we can find topological disks $B_{n}$ in $\mathbb{C} \backslash\{z$ : Dist $(z, D) \leq K / 4\}$ containing $E^{m_{n}}\left(x_{n}\right)$ and $E^{m_{n}}\left(y_{n}\right)$ with the following property:

$$
\frac{\operatorname{Diam} B_{n}}{\operatorname{Dist}\left(B_{n}, \infty\right)} \leq \frac{2}{C}
$$


and such that $B_{n}=p_{n}(\mathbb{D})$, where $\mathbb{D}$ is the unit disk and the distortion of the map $p_{n}$ is bounded by a constant which does not depend on $n$. The preimage of $B_{n}$ is contained in the union of the exponential tracts. Let $W_{n}$ be the component of $E^{-m_{n}}\left(B_{n}\right)$ containing $x_{n}$ and $y_{n}$. Using the spherical version of the Koebe Distortion Theorem ([10, Lemma 1.2]) we find that for any branch $H$ of $E^{-m_{n}}$ the distortion of $H$ on $B_{n}$ is bounded independently of $n$ and of the choice of $H$. If $n$ is large enough, say $n \geq n_{0}$, then $x, y \in W_{n}$ and $U=\bigcap_{n \geq n_{0}} W_{n}$ contains an open set $V$ such that $x, y \in V$.

The family $E^{m_{n}}: V \rightarrow B_{n}$ is normal. This is a contradiction, as $x, y \in$ $J(E)$. Hence there cannot be two limit points for $\beta$.

CASE II: $t_{n} \nrightarrow \infty$. If $t_{n}$ contains an unbounded sequence we are back in case I. So assume that the sequence $t_{n}$ is bounded. Then there exists $m_{1} \geq 0$ such that

$$
\min \left\{\operatorname{Dist}\left(E^{m_{1}}\left(x_{n}\right), \infty\right), \operatorname{Dist}\left(E^{m_{1}}\left(y_{n}\right), \infty\right)\right\} \geq C \operatorname{Dist}\left(E^{m_{1}}\left(x_{n}\right), E^{m_{1}}\left(y_{n}\right)\right)
$$

for infinitely many $n$, and hence

$$
\min \left\{\operatorname{Dist}\left(E^{m_{1}}(x), \infty\right), \operatorname{Dist}\left(E^{m_{1}}(y), \infty\right)\right\} \geq C \operatorname{Dist}\left(E^{m_{1}}(x), E^{m_{1}}(y)\right) .
$$

Now consider $E^{m_{1}+1}(\beta)$. It has (at least) two limit points: $E^{m_{1}+1}(x)$ and $E^{m_{1}+1}(y)$. We can find two sequences $x_{n}^{1}, y_{n}^{1}$ of points in $E^{m_{1}+1}(\beta)$ lying in reverse order such that

$$
x_{n}^{1} \rightarrow E^{m_{1}+1}(x), \quad y_{n}^{1} \rightarrow E^{m_{1}+1}(y) .
$$

This means that there exists a sequence $t_{n}^{1}$ such that $E^{t_{n}^{1}}\left(x_{n}^{1}\right) \prec E^{t_{n}^{1}}\left(y_{n}^{1}\right)$. If $t_{n}^{1}$ contains a subsequence tending to $\infty$ we are in case I. Otherwise we can find $m_{2}$ such that

$$
\min \left\{\operatorname{Dist}\left(E^{m_{2}}\left(x_{n}^{1}\right), \infty\right), \operatorname{Dist}\left(E^{m_{2}}\left(y_{n}^{1}\right), \infty\right)\right\} \geq C \operatorname{Dist}\left(E^{m_{2}}\left(x_{n}^{1}\right), E^{m_{2}}\left(y_{n}^{1}\right)\right)
$$

for infinitely many $n$, and hence

$$
\begin{aligned}
\min \left\{\operatorname { D i s t } \left(E^{m_{2}}\left(E^{m_{1}+1}(x)\right)\right.\right. & \left., \infty), \operatorname{Dist}\left(E^{m_{2}}\left(E^{m_{1}+1}(y)\right), \infty\right)\right\} \\
& \geq C \operatorname{Dist}\left(E^{m_{2}}\left(E^{m_{1}+1}(x)\right), E^{m_{2}}\left(E^{m_{1}+1}(y)\right)\right) .
\end{aligned}
$$

We now proceed by induction. Assume that we have integers $m_{1}, \ldots, m_{k} \geq 0$ such that

$$
\begin{gathered}
\min \left\{\operatorname{Dist}\left(E^{m_{1}+1+m_{2}+1+\ldots+m_{k}}(x), \infty\right), \operatorname{Dist}\left(E^{m_{1}+1+m_{2}+1+\ldots+m_{k}}(y), \infty\right)\right\} \\
\geq C \operatorname{Dist}\left(E^{m_{1}+1+m_{2}+1+\ldots+m_{k}}(x), E^{m_{1}+1+m_{2}+1+\ldots+m_{k}}(y)\right) .
\end{gathered}
$$

Consider $E^{m_{1}+1+\ldots+m_{k}+1}(\beta)$. We find two sequences $x_{n}^{k}, y_{n}^{k}$ of points in $E^{m_{1}+\ldots+m_{k}+1}(\beta)$ lying in reverse order such that

$$
x_{n}^{k} \rightarrow E^{m_{1}+m_{2}+1+\ldots+m_{k}+1}(x), \quad y_{n}^{k} \rightarrow E^{m_{1}+1+m_{2}+1+\ldots+m_{k}+1}(y) .
$$


So we can find $m_{k+1}$ such that

$$
\begin{aligned}
\min \left\{\operatorname{Dist}\left(E^{m_{k+1}}\left(x_{n}^{k}\right), \infty\right), \operatorname{Dist}\left(E^{m_{k+1}}\left(y_{n}^{k}\right), \infty\right)\right\} \\
\\
\geq C \operatorname{Dist}\left(E^{m_{k+1}}\left(x_{n}^{k}\right), E^{m_{k+1}}\left(y_{n}^{k}\right)\right)
\end{aligned}
$$

for infinitely many $n$ (if such an $m_{k+1}$ does not exist we are in case I). Hence $\min \left\{\operatorname{Dist}\left(E^{m_{1}+1+\ldots+m_{k}+1+m_{k+1}}(x), \infty\right), \operatorname{Dist}\left(E^{m_{1}+1+\ldots+m_{k}+1+m_{k+1}}(y), \infty\right)\right\}$

$$
\geq C \operatorname{Dist}\left(E^{m_{1}+1+\ldots+m_{k}+1+m_{k+1}}(x), E^{m_{1}+1+\ldots+m_{k}+1+m_{k+1}}(y)\right) .
$$

Let $B_{m_{k}}$ be a topological disk in $\mathbb{C} \backslash\{z: \operatorname{Dist}(z, D) \leq K / 4\}$ containing $E^{m_{1}+1+\ldots+m_{k}}(x)$ and $E^{m_{1}+1+\ldots+m_{k}}(y)$ with

$$
\frac{\operatorname{Diam} B_{m_{k}}}{\operatorname{Dist}\left(B_{m_{k}}, \infty\right)} \leq \frac{2}{C}
$$

and such that each $B_{m_{k}}$ is the image of the unit disk under a map whose distortion is bounded independently of $m_{k}$. Let $W_{m_{k}}$ denote the component of $E^{-\left(m_{1}+1+\ldots+m_{k}\right)}\left(B_{m_{k}}\right)$ containing $x$ and $y$. Since the distortion of the branches of $E^{-\left(m_{1}+1+\ldots+m_{k}\right)}$ is bounded on each $B_{m_{k}}$ the set $\bigcap_{k=1}^{\infty} W_{m_{k}}$ contains an open set $U$. The family $\left\{E^{m_{1}+1+\ldots+m_{k}}\right\}_{k=1}^{\infty}$ is normal on $U$ and we get a contradiction. Therefore $\beta$ cannot have two limit points.

Acknowledgments. The author would like to thank Prof. Feliks Przytycki for his helpful suggestions.

\section{References}

[1] W. Bergweiler, Iteration of meromorphic functions, Bull. Amer. Math. Soc. 29 (1993), 151-188.

[2] W. Bergweiler, M. Haruta, H. Kriete, H.-G.Meier and N. Terglane, On the limit functions of iterates in wandering domains, Ann. Acad. Sci. Fenn. Ser. A I Math. 18 (1993), 369-375.

[3] R. L. Devaney and L. Goldberg, Uniformization of attracting basins for exponential maps, Duke Math. J. 2 (1987), 253-266.

[4] R. L. Devaney and M. Krych, Dynamics of $\exp (z)$, Ergodic Theory Dynam. Systems 4 (1984), 35-52.

[5] R. L. Devaney and F. Tangerman, Dynamics of entire functions near the essential singularity, ibid. 6 (1986), 489-503.

[6] A. E. Eremenko and M. Lyubich, Dynamical properties of some classes of entire functions, Ann. Inst. Fourier (Grenoble) 42 (1992), 989-1020.

[7] M. V. Jakobson, Markov partitions for rational endomorphisms of the Riemann sphere, in: Multicomponent Random Systems, Adv. Probab. Related Topics 6, Dekker, New York, 1980, 381-396.

[8] B. Karpińska, Area and Hausdorff dimension of the set of accessible points of the Julia sets of $\lambda \exp z$ and $\lambda \sin z$, Fund. Math. 159 (1999), 269-287.

[9] -, Hausdorff dimension of the hairs without endpoints for $\lambda \exp z$, C. R. Acad. Sci. Paris Sér. I 328 (1999), 1039-1044.

[10] F. Przytycki, Iterations of holomorphic Collet-Eckmann maps: conformal and invariant measures, Trans. Amer. Math. Soc. 350 (1998), 717-742. 
[11] F. Przytycki, M. Urbański and A. Zdunik, Harmonic, Gibbs and Hausdorff measures for holomorphic maps, Ann. of Math. 130 (1989), 1-40.

[12] G. Stallard, The Hausdorff dimension of Julia sets of entire functions, Ergodic Theory Dynam. Systems 11 (1991), 769-777.

[13] - , The Hausdorff dimension of Julia sets of entire functions III, Math. Proc. Cambridge Philos. Soc. 122 (1997), 223-244.

Faculty of Mathematics and Information Sciences

Technical University of Warsaw

Pl. Politechniki 1

00-661 Warszawa, Poland

E-mail: bkarpin@impan.gov.pl 